\section{Case Reports in Dermatology}

\title{
Giant Congenital Melanocytic Nevi: An Update and Emerging Therapies
}

\author{
Girish Gulab Meshram $^{a} \quad$ Neeraj Kaur ${ }^{b} \quad$ Kanwaljeet Singh Hura ${ }^{c}$ \\ ${ }^{a}$ Department of Pharmacology, Postgraduate Institute of Medical Education and Research \\ and Dr. Ram Manohar Lohia Hospital, New Delhi, India; ${ }^{b}$ Department of Radiology, The \\ University of Texas Health Science Center, San Antonio, TX, USA; 'Department of \\ Pediatrics, Richmond University Medical Center, New York, NY, USA
}

\section{Keywords}

Giant congenital melanocytic nevi · Neurocutaneous melanosis · Malignant melanoma

\begin{abstract}
Giant congenital melanocytic nevi (GCMN) are a rare occurrence. Gain-of-function mutation in the NRAS gene is found to be associated with GCMN, causing abnormal proliferation of embryonic melanoblasts. The two major complications associated with GCMN are malignant melanoma and neurocutaneous melanosis. Treatment of GCMN has conventionally been surgical. However, the role of NRAS inhibitors and inactivation of nevus tissue by high hydrostatic pressure are being explored. We present a case of a 1-day-old neonate born with GCMN, along with a review of the literature.

(C) 2018 The Author(s)

Published by S. Karger AG, Basel
\end{abstract}

\section{Introduction}

Congenital melanocytic nevi (CMN) are large brown-to-black skin lesions caused due to genetic mutations which lead to defective proliferation/differentiation/migration of melanoblasts. Melanoblasts are precursor cells of melanocytes [1, 2]. Giant CMN (GCMN) with a 
diameter greater than $20 \mathrm{~cm}$ have an estimated incidence of between 1/20,000 and $1 / 500,000$ births. The risk of transformation of GCMN to malignant melanoma varies between 0 and 3.8\% [3]. Besides malignant transformation, patients with GCMN need to be periodically assessed for neurological abnormalities and psychosocial impairment. GCMN are usually managed surgically [4]. However, new chemotherapy-based therapies are being developed. We present a rare case of a 1-day-old neonate with GCMN, discussing its etiology and latest trends in management.

\section{Case Presentation}

The patient is a 1-day-old male neonate, who presented to the pediatric department with several black pigmented patches over his body. The mother of the patient was a primigravida, and gave birth to her child via normal vaginal delivery at term without any complications. The parents of the child did not have a consanguineous marriage. None of the close family members had similar skin lesions. On examination, an extensive pigmented nevus was observed encompassing the abdomen, pelvis, groin, upper thighs, buttocks, and the lumbosacral region of the back. Within the pigmented nevus there were several irregularly shaped macules, papules, and plaques of various colors. On palpation the surface of the nevus was irregular. Hypertrichosis was absent. Multiple similar smaller pigmented nevi of 2-5 $\mathrm{cm}$ in size were present on various other parts of the body and extremities (Fig. 1, 2). No other congenital anomaly was observed. Neurological examination was unremarkable. A clinical diagnosis of GCMN was made. Routine blood and urine examination did not reveal any abnormalities. Biopsy sample revealed findings consistent with GCMN without malignant changes. Transfontanellar ultrasonography and X-ray of the spine did not detect any abnormalities. The patient was advised frequent follow-up visits and an MRI scan of the brain and spine was scheduled after 2 months. However, the patient did not follow up with the hospital.

\section{Discussion}

CMN arise from gain-of-function somatic mutations in either BRAF at V600 or NRAS at Q6. These mutations affect the microtubule-associated protein kinase (MAPK) signal transduction pathway, leading to abnormal proliferation of embryonic melanoblasts [1]. Recent evidence suggests that $C d o n$, a regulator acting via the sonic hedgehog pathway, exacerbates the development of CMN in the context of an NRAS mutation by causing excessive release of endothelin-1 [5].

The proto-oncogenes c-met and c-kit, which regulate hepatocyte growth factor/scatter factor and stem cell factor, respectively, are found to play a role in the formation of CMN [6]. These mutations lead to aberrations in the development/migration of neuroectodermal cells present in the neural crest, from which melanoblasts arise. These abnormal melanoblasts scatter to various parts of the body, leading to nevi [2, 4, 6, 7]. CMN are predominantly caused by sporadic de novo mutations. However, some familial cases have also been reported $[1,4]$.

CMN are characterized by their projected adult size, number of smaller satellite lesions, anatomical localization, and degree of rugosity, hypertrichosis, nodularity, and color heterogeneity [8]. A nevus with a projected adult size greater than $40 \mathrm{~cm}$ is classified as GCMN, as 
in our case. GCMN are further classified into six categories according to their anatomical location as bolero, back, bathing-trunk, breast/belly, body extremity, and body [8]. Our patient belonged to the bathing-trunk category as the nevus was present in the region of the genitals and buttocks without extension to the shoulder or neck region.

Biopsy examination of our patient revealed increased melanocytes in the epidermis, dermis, and skin appendages, characteristic of CMN [4, 6]. GCMN are posed at a significantly greater risk of transforming into malignant melanomas, as compared to smaller-sized nevi $[2,4,6,9]$. Furthermore, GCMN presenting in the bathing-trunk pattern, as in our patient, are associated with an increased risk of malignant transformation [8]. Analysis of proliferative markers such as Mib-1, p16, p21, p27, and p53 may aid in early detection of malignant changes [7].

Another complication associated with GCMN is neurocutaneous melanosis (NCM). NCM is characterized by abnormal melanosis of the central nervous system. NCM is more common in patients with GCMN encompassing the head, neck, or paravertebral area (bathingtrunk nevi), or GCMN with several satellite nevi $[2,6,7]$. Most patients with NCM are asymptomatic at birth. NCM may manifest as hydrocephalus, lethargy, seizures, cranial nerve palsy, developmental delays, headache, and neuropsychiatric symptoms [2, 4, 7]. It has been found that patients with NCM have high urine dopamine levels, which could serve as a useful prognostic marker [2]. Although our patient was asymptomatic, he was at a risk of NCM. Hence, the parents of our patient were provided guidance for detecting signs and symptoms of NCM, and were requested to follow up with MRI scans of the head and spine.

Management of GCMN is symptomatic and palliative. Surgical techniques for GCMN include serial resection, excision followed by skin grafts/substitutes, and use of tissue expanders followed by resection $[2,4]$. Decision for surgery is based on the location/size of the nevi, risk of malignancy/NCM, psychological implications, and cosmetic concerns [4, 7]. Nonexcisional techniques for GCMN include dermabrasion, laser ablation, curettage, and chemical peel [4].

A novel surgical technique showing promising results in GCMN is full-thickness excision of the nevus tissue, followed by its inactivation by high hydrostatic pressure (200 MPa for 10 $\mathrm{min}$ ) and replanting it to its original site $[9,10]$. However, more clinical trials with a larger sample size and longer follow-up periods are needed to determine the success rate of this technique. The use of an NRAS inhibitor (trametinib), targeting the MAPK pathway, is proposed for treating patients with GCMN associated with an underlying NRAS mutation $[1,2]$. However, clinical studies to substantiate this claim are yet to be conducted. Use of endothelin-1 receptor antagonists has been proposed as a therapeutic approach for GCMN, based on evidence generated from preclinical studies [5].

\section{Conclusions}

GCMN, although a rare condition, is associated with severe life-threatening complications such as malignant melanoma and NCM. The first-line approach for GCMN is excisionbased surgery. As the molecular and genetic basis of GCMN is not entirely understood, the development of chemotherapeutic agents targeting candidate receptors may take several years. 


\section{Statement of Ethics}

Written informed consent was obtained from the parents of the patient for publication.

\section{Disclosure Statement}

The authors declare that there are no conflicts of interest regarding the publication of this paper.

\section{References}

1 Etchevers HC: Hiding in plain sight: molecular genetics applied to giant congenital melanocytic nevi. J Invest Dermatol 2014;134:879-882.

$\longrightarrow 2$ Recio A, Sanchez-Moya AI, Felix V, Campos Y: Congenital melanocytic nevus syndrome: a case series. Actas Dermosifiliogr 2017;108:e57-e62.

3 Tonseth KA, Filip C, Hermann R, Vindenes H, Hogevoid HE: Extraordinary large giant congenital nevus treated with Integra dermal regeneration template. Plast Reconstr Surg Glob Open 2015;10:e469.

4 Marchesi A, Leone F, Sala L, Gazzola R. Vaienti L. Giant congenital melanocytic naevi: a review of literature. Ped Med Chir 2012;34:73-76.

-5 Chitsazan A, Ferguson B, Villani R, Handoko HY, Mukhopadhyay P, Gabrielli B, Mooi WJ, Soyer HP, Lambie D, Khosrotehrani K, Morahan G, Walker GJ: Keratinocyte sonic hedgehog up-regulation drives the development of giant congenital nevi via paracrine endothelin-1 secretion. J Invest Dermatol 2017, Epub ahead of print. http://www.jidonline.org/article/S0022-202X(17)33158-5/pdf.

-6 Viana ACL, Gojinto B, Bittencourt FV: Giant congenital melanocytic nevus. An Bras Dermatol 2013;88:863-878.

7 Chien JC, Niu DM, Wang MS, Liu MT, Lirng JF, Chen SJ, Hwang B: Giant congenital melanocytic nevi in neonates: report of two cases. Pediatr Neonatol 2010;51:61-64.

-8 Martins da Silva VP, Marghoob A, Pigem R, Carrera C, Aguilera P, Puig-Butille JA, Malvehy J: Patterns of distribution of giant congenital melanocytic nevi (GCMN): the 6B rule. J Am Acad Dermatol 2017;76:689-694.

9 Sakamoto M, Morimoto N, Jinno C, Mahara A, Ogino S, Suzuki S, Kusumoto K, Yamaoka T: Melanin pigments in the melanocytic nevus regress spontaneously after inactivation by high hydrostatic pressure. PLoS One 2017;12:e0186958.

10 Morimoto N, Jinno C, Sakomoto M, Kakudo N, Yamaoka T, Kusumoto K: An exploratory clinical trial of a novel treatment for giant congenital melanocytic nevi combining inactivated autologous nevus tissue by high hydrostatic pressure and cultured epidermal autograft: study protocol. JMIR Res Protoc 2016;5:e162. 


\section{Case Reports in Dermatology}

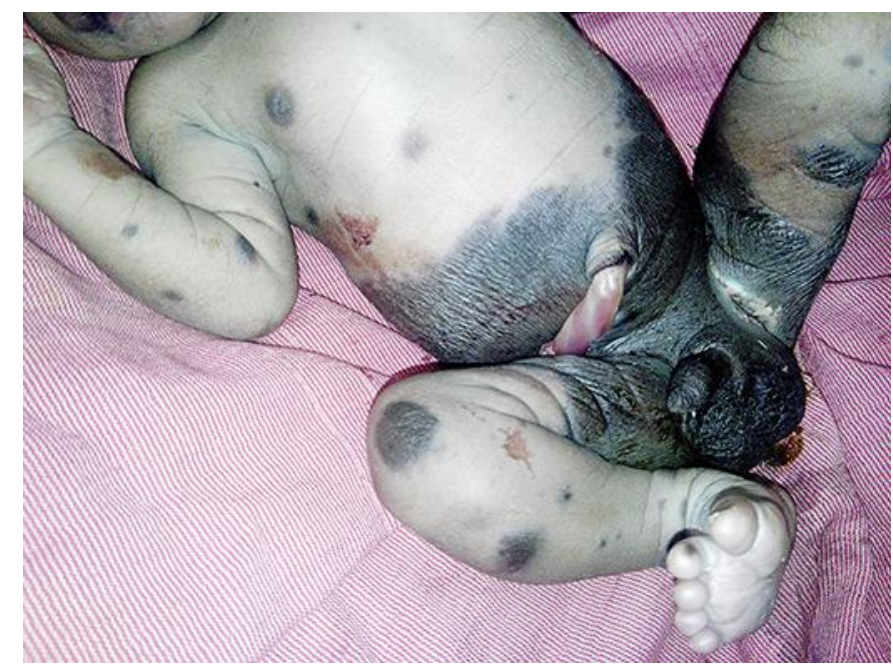

Fig. 1. Giant nevus encompassing the lower abdomen, genital area, and upper thighs.

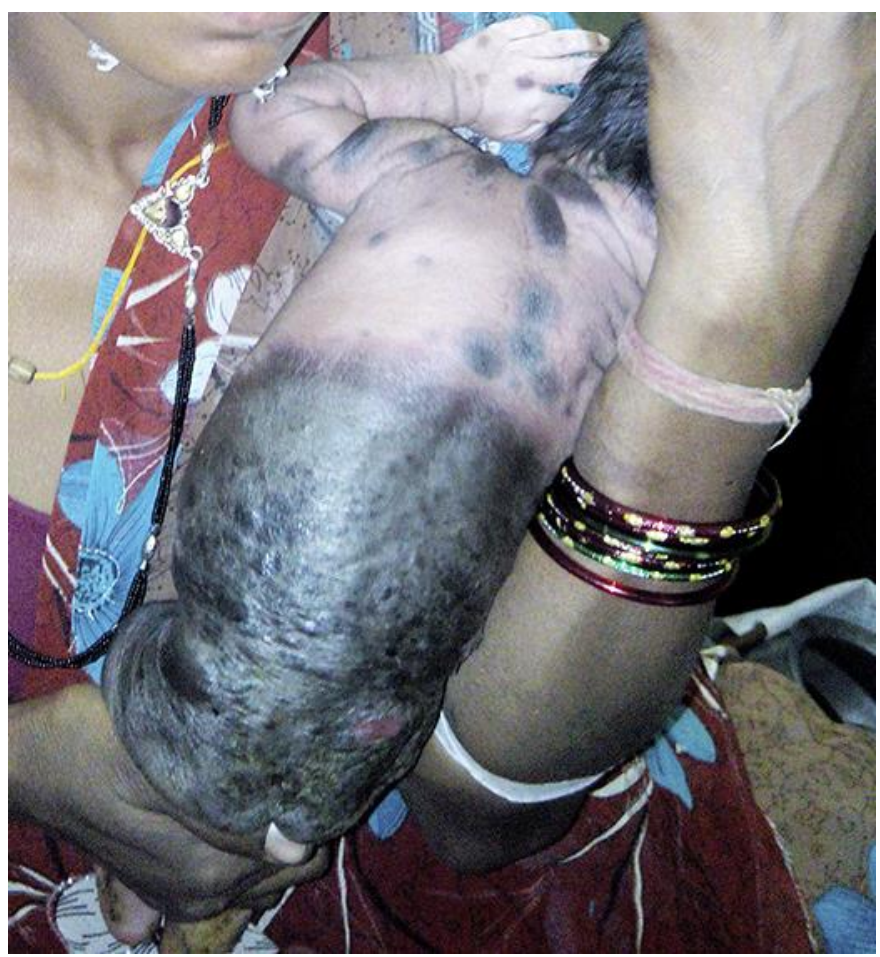

Fig. 2. Giant nevus involving the middle and lower back, gluteal region, and upper thighs. Numerous smaller satellite nevi are also observed on the neck, upper back, and upper limbs. 